\title{
Tödliche Pneumonitis unter Basistherapie mit Methotrexat
}

\author{
Martos, V P ; Vonlanthen, B
}

\begin{abstract}
We present the case of a 71 year old Swiss woman with a symptomatic rheumatic arthritis whose rheumatologist started a therapy with methotrexate. While the symptoms disappeared completely, the woman died about two months later suddenly. By then she got a cumulative dose of $160 \mathrm{mg}$. The forensic post-mortem examination showed a fibrosing alveolitis. If respiratory symptoms appear under methotrexat regimen therapy, immediate stopping the medication could be life-saving. In accordance to the chest x-ray a cortisone therapy can be established.
\end{abstract}

DOI: https://doi.org/10.1007/s00393-011-0918-z

Other titles: Fatal pneumonitis during low-dose methotrexate treatment

Posted at the Zurich Open Repository and Archive, University of Zurich ZORA URL: https://doi.org/10.5167/uzh-64728

Journal Article

Published Version

Originally published at:

Martos, V P; Vonlanthen, B (2012). Tödliche Pneumonitis unter Basistherapie mit Methotrexat. Zeitschrift für Rheumatologie, 71(1):75-77.

DOI: https://doi.org/10.1007/s00393-011-0918-z 
Z Rheumatol 2012 $\cdot 71: 75-77$

DOI 10.1007/s00393-011-0918-z

(C) Springer-Verlag 2012

\section{Redaktion}

E. Reinhold-Keller, Hamburg

F. Moosig, Lübeck/Bad Bramstedt
V.P. Martos · B. Vonlanthen

Institut für Rechtsmedizin, Abteilung Forensische Medizin, Universität Zürich

\section{unter Basistherapie mit Methotrexat}

\section{Anamnese}

Eine 71-jährige, verheiratete Frau suchte anfangs März 2009 ihren Hausarzt auf wegen kürzlich aufgetretenen Beschwerden wie bis zu $2 \mathrm{~h}$ lang anhaltender Morgensteifigkeit und schmerzenden, geschwollenen Hand- und Fingergelenken. In den letzten Monaten sei es zu einem Gewichtsverlust von $5 \mathrm{~kg}$ gekommen. Die pensionierte Verkäuferin litt seit etwa 20 Jahren an Verdickungen der Fingerendgelenke (Bouchard- und HeberdenArthrose), die gelegentlich Schmerzen bereitet haben und früher vom niedergelassenen Rheumatologen mit Chondroitinsulfat behandelt worden waren.

Anamnestisch bestanden eine arterielle Hypertonie und ein Status nach Cholezystektomie und abdomineller Hysterektomie mit Adnexektomie.

\section{Klinischer Befund, Diagnostik, Therapie und Verlauf}

\section{Erste Konsultationen}

Der Hausarzt stellte in der Untersuchung keine wesentliche Gelenkentzündung fest. Labormäßig betrug die BSR $60 \mathrm{~mm} / \mathrm{h}$ und

\begin{tabular}{|l|l|}
\hline \multicolumn{2}{|l}{ Abkürzungen } \\
\hline Anti-CCP & $\begin{array}{l}\text { Antikörper gegen cyclisches } \\
\text { citrulliniertes Peptid }\end{array}$ \\
\hline$B S R$ & Blutsenkungsreaktion \\
\hline$C R P$ & C-reaktives Protein \\
\hline$M C P$ & Metacarpo-Phalangealgelenk \\
\hline$M T X$ & Methotrexat \\
\hline$P I P$ & Proximales Interphalagealgelenk \\
\hline
\end{tabular}

das CRP $51 \mathrm{mg} / \mathrm{l}$. Er vermutete eine aktivierte Polyarthrose und begann eine symptomatische Therapie mit Mefenaminsäure. Bei der nächsten Konsultation vermutete er eine rheumatoide Arthritis (RA) und verabreichte Diclofenac und Dexamethason i.m., was für rund 1 Tag Linderung brachte. Bei erneuter Vorstellung der Patientin rezeptierte er $20 \mathrm{mg}$ Prednisolon pro Tag p.o. und überwies sie zum früheren Rheumatologen.

\section{Überweisung}

Der Rheumatologe stellte Mitte März aufgrund der vorliegenden Symptomatik (Schmerzen in den PIP, MCP, im rechten Handgelenk und in den Schultergelenken) und den durchgeführten Laboruntersuchungen (positiv für den Rheumafaktor und Anti-CCP) die Diagnose einer seropositiven RA. Radiologisch lag eine Fingerpolyarthrose, eine geringgradige Rhiz- und Omarthrose vor. Zusätzlich zum Kortisonpräparat wurde eine Basistherapie mit wöchentlichen i.m.-Injektionen von $20 \mathrm{mg}$ Methotrexat (MTX) vorerst für 3 Monate installiert. Prophylaktisch erhielt die Patientin täglich $1 \mathrm{mg}$ Folsäure.

\section{Kontrolltermin}

Ende April 2009 war die Patientin bezüglich der Gelenkentzündung asymptomatisch. Der Faustschluss war kräftig. Laboranalytisch waren die Entzündungszeichen regredient (BSR $18 \mathrm{~mm} / \mathrm{h}, \mathrm{CRP}$ $15 \mathrm{mg} / \mathrm{l})$. Die Kortisondosis konnte auf $10 \mathrm{mg}$ täglich reduziert werden. Gegen aphthöse Beschwerden enoral gab der Rheumatologe Hexetidinlösung. Eine erneute Konsultation war für einen $3 \mathrm{Mo-}$ nate späteren Zeitpunkt geplant. Die Behandlung mit MTX sollte für mindestens 1 Jahr weitergeführt werden.

\section{Letzte Konsultation}

Am 1. Mai 2009 suchte die Patientin erneut ihren Hausarzt auf. Sie klagte über Schüttelfrost in der Nacht mit „Kollaps“ in der Küche und quälenden Reizhusten mit farblosem Auswurf. Bei fraglichen Rasselgeräuschen in der Lungenauskultation und erhöhten Entzündungswerten im Blut (Leukozyten 8,6 G/l, Neutrophile $84,4 \%$, CRP $56 \mathrm{mg} / \mathrm{l}$, BSR $22 \mathrm{~mm} / \mathrm{h}$ ) interpretierte der Hausarzt die Beschwerden am ehesten als Atemwegsinfekt und begann eine antibiotische Therapie mit $250 \mathrm{mg}$ Clarithromycin p.o. morgens und abends. Es wurde ein Kontrolltermin 3 Tage später vereinbart.

Noch bevor sie den Kontrolltermin hätte wahrnehmen können, kollabierte die Frau beim Einsteigen zu einer Autofahrt in den Pkw und wurde bewusstlos. Die durch die alarmierte Rettungssanität durchgeführte Reanimation war ohne Erfolg.

\section{Obduktionsbefunde}

In der an die Legalinspektion anschließenden, durch die Untersuchungsbe-

Zur besseren Lesbarkeit wurden die männlichen Personenbezeichnungen verwendet, gelten aber sinngemäß für beide Geschlechter. 


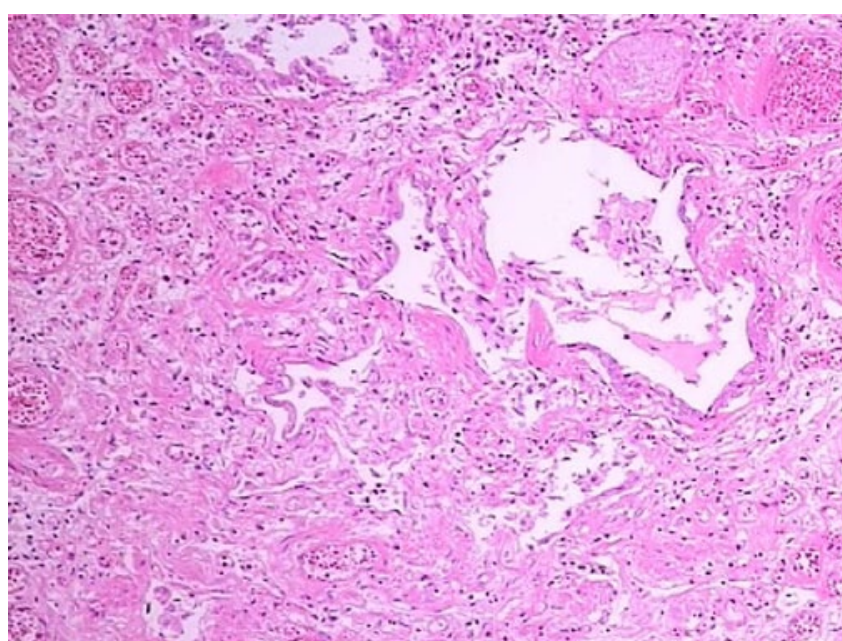

Abb. $1 \Delta$ Lungengewebe mit Fibrose und Zeichen einer Vaskulitis (40-fache Vergrößerung)

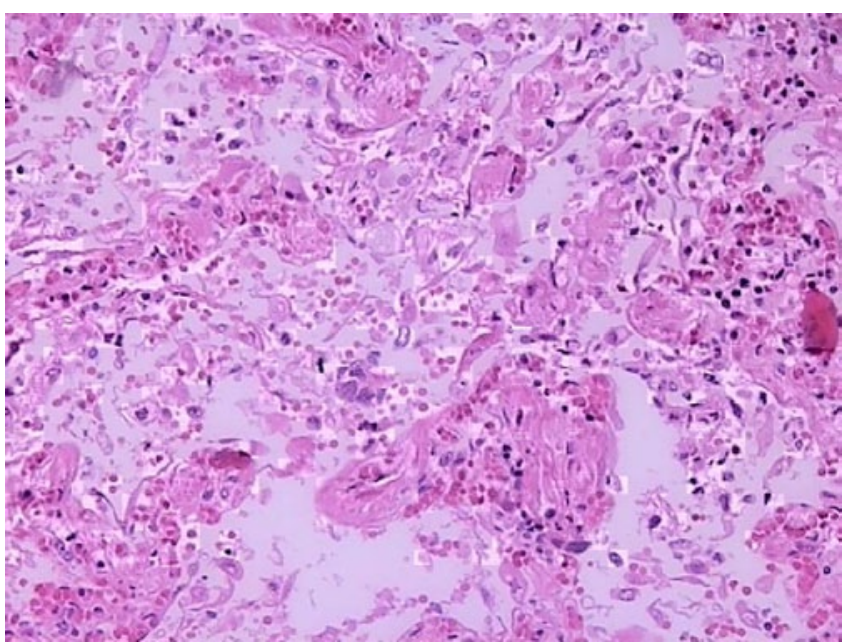

Abb. $2 \Delta$ Lungengewebe mit einem Granulom und Ausbildung von hyalinen Membranen (400-fache Vergrößerung) hörde angeordneten rechtsmedizinischen Obduktion konnten ein akutes Lungenödem und eine derbe Konsistenz des Lungengewebes festgestellt werden. Mikroskopisch zeigte sich eine chronische, überwiegend lymphozytäre, fibrosierende Pneumonitis. Arealweise waren im Lungenparenchym granulomatöse Formationen vorhanden. Es bestanden eine entzündliche Beteiligung der Gefäße (Vaskulitis) und eine partielle Sklerose der Lymphknoten

(• Abb. 1, 2). Sodann war eine chronische Bronchitis evident.

Die Autopsie der übrigen Organe wies eine hochgradige Sklerose aller Arterien nach. Am Herz fanden sich eine linksbetonte, exzentrische Hypertrophie, eine mittelgradige Koronarsklerose und eine feinfleckige Myokardfibrose. Die Leber war mittelgradig überwiegend grobtropfig verfettet und ohne entzündliche Veränderungen.

\section{Diskussion}

MTX ist ursprünglich ein Chemotherapeutikum, welches im Stoffwechsel als Antagonist der Folsäure wirkt. Durch einen intrazellulären Mangel an Folsäure bzw. ihrer Metaboliten kommt es zu einer Hemmung der Zellreproduktion. $\mathrm{Zu}$ Beginn wurde es ausschließlich antineoplastisch eingesetzt; im Verlauf wurde zunehmend die antiinflammatorische Wirkung der niedrigen Dosierung genutzt [2]. Seit den frühen 80er-Jah- ren wird es bei RA eingesetzt und hat sich als eigentlicher Goldstandard etabliert [3].

Im geschilderten Fall suchte die Patientin mit rheumatologischen Beschwerden ihren Hausarzt auf. Bei der Verdachtsdiagnose einer RA überwies er sie an den Spezialisten. Dieser begann eine Therapie mit MTX, wodurch die synovitischen Beschwerden praktisch verschwanden. Außer einer Mukositis (aphthöse Stomatitis) waren im Verlauf keine Nebenwirkungen aufgetreten. Als die Patientin rund 2 Monate nach Beginn der MTX-Therapie mit Atem- und Kreislaufbeschwerden vorstellig wurde, ging der Hausarzt von einem Atemwegsinfekt aus und begann eine antibiotische Therapie.

Nachdem die Patientin 2 Tage später unerwartet verstorben war, wurde in der rechtsmedizinischen Obduktion eine fibrosierende Pneumonitis unter MTX festgestellt. Die kumulative Dosis betrug etwa $160 \mathrm{mg}$. Aufgrund der histologischen Befunde ist von einem sich seit Tagen bis Wochen entwickelnden, chronischen Lungenprozess auszugehen. Typisch für die Diagnose ist der Nachweis eines diffusen Alveolarwandschadens und interstitieller Infiltrate mit oder ohne Granulombildung [2]. Der initiale Verdacht auf Lungenthromboembolien konnte ausgeschlossen werden. Todesursächlich wurde ein Rechtsherzversagen wegen Erhöhung des Gefäßwiderstands im kleinen Kreislauf vermutet, bei einem gleichzeitig ar- teriosklerotisch vorgeschädigten Herzen.

Als Auslöser einer medikamenteninduzierten Pneumonitis (oder Pneumopathie) sind u. a. Zytostatika oder Herzmedikamente (z. B. Amiodaron-Pneumonitis) bekannt. Typische klinische Zeichen sind Dyspnoe, unproduktiver Husten und Fieber. Dabei kann die Pneumonitis unter der Therapie oder erst mit einer gewissen Latenz nach Absetzen der Medikation auftreten [2].

$\mathrm{Zu}$ Beginn der MTX-Therapie wurde kein Thorax-Röntgenbild angefertigt. Ein solches hätte vielleicht auch bei fehlender Anamnese und Klinik eine vorbestehende Lungenerkrankung aufdecken können, weshalb es zu empfehlen ist. Zudem erfolgten bei der Patientin keine serologischen Kontrollen während der Basistherapie mit MTX. Vielleicht hätten sich dadurch bereits beim Auftreten von Aphthen Hinweise auf weitere Nebenwirkungen ergeben.

Die parenterale Verabreichung, wie in unserem Fall erfolgt, ist wegen der größeren Hemmschwelle einer Dosissteigerung unseres Erachtens empfehlenswert. Neben der pulmonalen Manifestation kann MTX vor allem bei Überdosierung u. a. zu Vaskulitis, Myelosuppression mit Panzytopenie, Leberzellnekrosen und Leberzirrhose führen [3]. 


\section{Differenzialdiagnose}

Im dargestellten Verlauf traten die pulmonalen Veränderungen unter einer niedrigdosierten Therapie mit MTX auf. Differenzialdiagnostisch denkbar sind natürlich auch die direkten toxischen Wirkungen durch meist akzidentelle Überdosierungen mit MTX [5] und eine durch Herzmedikamente wie z. B. Amiodaron induzierte Pneumonitis.

\section{Fazit für die Praxis}

Treten unter der Therapie mit MTX respiratorische Symptome auf, was meist in den ersten Monaten nach Beginn der Fall ist, muss die Medikamentengabe sofort gestoppt werden. In der Praxis kann wie zu Beginn der MTX-Gabe ein Thorax-Röntgenbild angefertigt werden und entsprechend dem Befund eine Therapie mit Kortison eingeleitet werden [4]. Oft sind den Kollegen anderer Fachrichtungen die Nebenwirkungen rheumatologischer Spezifika nicht geläufig [1, 5]. Eine Instruktion der Patienten zu Beginn der Therapie ist notwendig und die Abgabe eines Patientenausweises zusätzlich hilfreich.

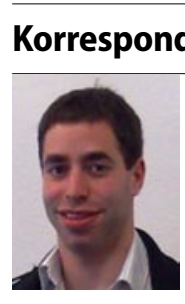

Institut für Rechtsmedizin, Abteilung Forensische Medizin, Universität Zürich

Winterthurerstr. 190/52,

CH-8057 Zürich

Schweiz

viktor.martos@irm.uzh.ch

Danksagung. Wir bedanken uns bei Prof. Dr. med Alan Tyndall, Chefarzt Rheumatologische Universitätsklinik, Felix-Platter-Spital Basel, für die kritische Durchsicht des Artikels vor Einreichung. Auf vorgängige Anfrage wurde durch die zuständigen Untersuchungsbehörden die anonymisierte Fallpräsentation genehmigt.

Interessenkonflikt. Der korrespondierende Autor gibt an, dass kein Interessenkonflikt besteht.

\section{Literatur}

1. Fässler EMI, Galeazzi RL (2003) Low-dose-Methotrexat: Nebenwirkungen und Toxizität. Schweiz Med Forum 49:1211-1215

2. Imokawa S, Colby TV, Leslie KO, Helmers RA (2000) Methotrexate pneumonitis: review of the literature and histopathological findings in nine patients. Eur Respir J 15:373-381

3. Moisa A, Fritz P, Benz D, Wehner HD (2006) latrogenically-related, fatal methotrexate intoxication: a series of four cases. Forensic Sci Int 156:154-157

4. Nigg L, Caspar C, Imhof E (2003) Pneumonitis unter niedrig dosierter Methotrexat-Therapie. Schweiz Med Forum 40:964-967

5. Stäubli M (2009) Latrogene Methotrexat-Intoxikation. Schweiz Med Forum 40:706
Z Rheumatol $2012 \cdot 71: 75-77$

DOI 10.1007/s00393-011-0918-z

(C) Springer-Verlag 2012

\section{V.P. Martos · B. Vonlanthen}

\section{Tödliche Pneumonitis unter Basistherapie mit Methotrexat}

Zusammenfassung

Wir schildern den Fall einer 71-jährigen Schweizerin mit symptomatischer rheumatoider Arthritis, welche vom Rheumatologen mit Methotrexat behandelt worden war. Die Therapie sprach sehr gut an, jedoch starb die Frau unverwartet 2 Monate später bei einer kumulativen Dosis von 160 mg. In der rechtsmedizinischen Obduktion konnte eine fibrosierende Pneumonitis festgestellt werden. Solch ein fataler Verlauf könnte durch sofortiges Absetzen des Methotrexats bei ersten respiratorischen Symptomen sowie durch Diagnostik mittels Thoraxröntgen und entsprechende Kortisongabe womöglich verhindert werden.

Schlüsselwörter

Methotrexat $\cdot$ Pneumonitis $\cdot$ Rheumatoide Arthritis · Tödlich · Vorsichtsmaßnahmen

\section{Fatal pneumonitis during low-dose methotrexate treatment}

Abstract

We present the case of a 71 year old Swiss woman with a symptomatic rheumatic arthritis whose rheumatologist started a therapy with methotrexate. While the symptoms disappeared completely, the woman died about two months later suddenly. By then she got a cumulative dose of $160 \mathrm{mg}$. The forensic post-mortem examination showed a fibrosing alveolitis. If respiratory symptoms appear under methotrexat regimen therapy, immediate stopping the medication could be lifesaving. In accordance to the chest $\mathrm{x}$-ray a cortisone therapy can be established.

Keywords

Methotrexate · Pneumonitis · Rheumatoid Arthritis · Lethal · Precautions 